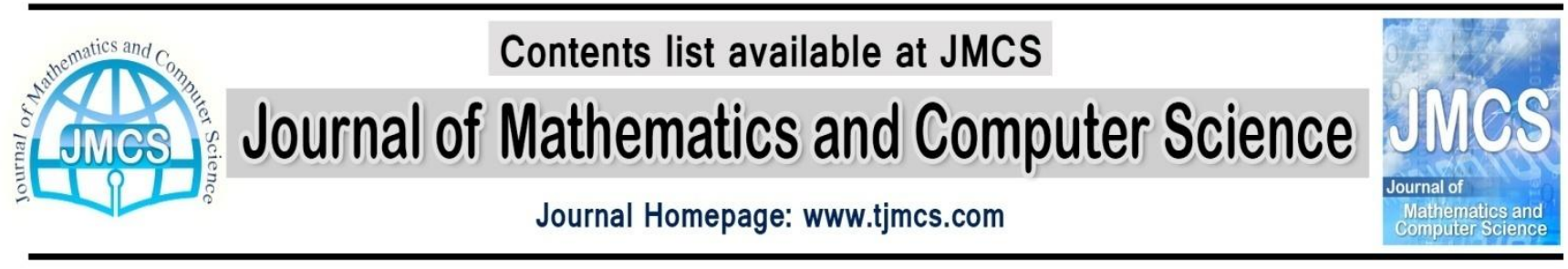

\title{
Performance Analysis of Manufacturing Systems Using Deterministic and Stochastic Petri Nets
}

\author{
Hassan Haleh ${ }^{1}$, Arman Bahari ${ }^{2, *}$, Behnoosh Moody ${ }^{3}$ \\ ${ }^{l}$ Department of Industrial Engineering, AssistantProfessor, Golpayegan University of Technology, \\ Golpayegan, Iran \\ ${ }^{2}$ Department of Industrial Engineering, Islamic Azad University, Science and Research Branch, \\ Tehran, Iran \\ ${ }^{3}$ Department of Management, University of Sistan \& Baluchistan, Sistan \& Baluchistan,Iran
}

"arman_bahari@eng.usb.ac.ir

Article history:

Received April 2014

Accepted May 2014

Available online May 2014

\begin{abstract}
Most of manufacturing industries in our country practice the traditional production systems. Effective management of the steady state operation is no longer enough to ensure the survival,let alone the successof an organization. The performance of the operations has to be improved continually in all its aspects, and it is driven by the quest for increased productivity, flexibility and continuously changing competitive environment.The increasing global character of market for goods and services, is stimulated by the factors like improvements in transport, data communication systems, and primarily the automation of manufacturing operations. These features need for continuous performance analysis and improvement of manufacturing systems. Therefore, the key to stay at the apex of global competition is to meet the dynamically changing need of customers. Manufacturing systems performance analysis using Petri Nets(PN) is one of the promising tools employed for assessing. PN models are now common place within the sphere of performance modeling of manufacturing systems due to reasons like graphical and precise representation of system activities and models at various levels of detail and ability to capture the existence of concurrency,parallelism,resource constraints and process dependencies accurately. This paper, focuses on analyzing the performance of the manufacturing process of pars metal, one of the manufacturing industries in the country, using PN so as to evaluate various performance parameters such as utilization rate of machines, bottleneck detection, cycle time, and throughput rate of system under consideration and providing solutions and recommendations for the pitfalls and ramification for attaining the optimum productivity.
\end{abstract}

Keywords: Manufacturing System, Performance Analysis, Stochastic Petri Net, Time Petri Net 


\section{Introduction}

Whether it is a manufacturing-based or a service-based company, the key to stay at the apex of global competition is to meet the dynamically changing need of customer [14]. Most of manufacturing industries in our country practice the traditional production systems. Effective management of the steady state operation is no longer enough to ensure the survival, let alone the success, of an organization. The performance of the operations has to be improved continually in all its aspects, and it is driven by the quest for increased productivity, flexibility and continuously changing competitive environment.

The increasing global character of market for goods and services, is stimulated by the factors like improvements in transport, telecommunication, and data communication systems, the rapid technological advancement and primarily the automation of manufacturing operations to decrease product life, cost of manufacturing and dependable deliveries. These features of the competitive environment certainly point to the need for continuous performance analysis, modeling of manufacturing system. The primary goals of a manufacturing system are to minimize the work in process inventory (WIP) and maximize the system utilization and output rate. Hence, the traditional production systems of our industries should be relooked to make the best use of the pertinent available technology and resources for better utilization of equipment $s$ and machineries [2].

Manufacturing systems performance analysis using Petri nets is one of the promising tools employed for assessing performance and then taking the necessary measures for improvements of existing deficiencies.

Performance is often a central issue in the design, development, and configuration of systems. It is not always enough to know that systems work properly they must also work effectively.

There are numerous studies, e.g. in the areas of computer and telecommunication systems, manufacturing, military, health care, and transportation, that have shown that time, money, and even lives can be saved if the performance of a system is improved. Performance analysis studies are conducted to evaluate existing or planned systems, to compare alternative configurations, or to find an optimal configuration of a system.

Analytical models, such as Markovian models can provide exact results regarding the performance of a system. The results are exact, in that they are not estimates of the performance of the system. However, the results provided by analytical models may or may not be accurate, depending on the assumptions that have been made in order to create the model. In many cases it is difficult to accurately model industrial-sized systems with analytical models .

A Petri Net is one of several mathematical representations of discrete distributed systems. It has been originated from Carl Adam Petri's doctoral dissertation work on communication with automata in 1962 is generally considered as integrated tool and methodology in various aspects of manufacturing systems. As a modeling language, it graphically depicts the structure of a distributed system and a directed bipartite graph with annotations. Petri Nets have proven to be very useful in the modeling, analysis, simulation and control of manufacturing system [10].The following advantages make Petri Nets an appropriate and effective tool and technology in analysis of manufacturing systems:

- Ease of modeling characteristics of a complex industrial system: concurrency, asynchronous and synchronous features, conflicts, mutual exclusion, precedence relation, and system dead locks.

- Ability to generate supervisory control code directly from the graphical Petri Net representation.

- Ability to check the system for understandable properties such as deadlocks and capacity overflow.

- Status information that allows for real-time control, monitoring and error recovery of the manufacturing system. 
- Usefulness for scheduling because the Petri net model contains the system precedence relations as well as constraints on discrete event performance.

- Graphical model uses very few but powerful primitives making it easy to understand[14].

\section{Fundamentals of Petri Nets}

Mathematical modeling or synthesis of EMS is the first step to analyze, simulate, and control the operations of such systems. When concurrency and synchronization concepts become keys for study of modern manufacturing systems control, Petri nets provide a most straightforward tool to model these concepts. In addition, they can model conflicts, non determinism, time information, and resource-sharing environments [13].

A Petri net is a bipartite directed graph that contains places represented by circles; transitions represented by boxes or bars, and directed arcs connecting places to transitions and transitions to places. The movement of tokens represents the dynamic nature of the modeled system in a Petri net. A token, represented as a small dot, is placed in a circle to indicate that the state is active. The current location and distribution of tokens in a Petri net is called its marking. The marking of the Petri net defines the state of the system. A Petri net containing tokens is called a marked Petri net [1].

Table 1. Basic elements of Petri Nets

\begin{tabular}{|l|l|l|l|}
\hline Name & Symbol & Name & Symbol \\
\hline Place & 0 & Token & \\
\hline Arcs & $\longrightarrow$ & Marked place & \\
\hline Transition & \multicolumn{1}{|c|}{$\longrightarrow$} & Transition with input and output arcs & $\stackrel{\text { Onput }}{\longrightarrow}$ \\
\hline
\end{tabular}

\subsection{Formal definition of Petri Nets}

A black-and-white Petri net or ordinary PN can be formally defined as a four-tuple [4] : PN = (P, T, I, O) where:

$\mathrm{P}$ is a finite non-empty set of places. $\mathrm{P}=\{\mathrm{p} 1, \mathrm{p} 2, \ldots, \mathrm{pn}\}$

$\mathrm{T}$ is a finite non-empty set of transitions. $\mathrm{T}=\{\mathrm{t} 1, \mathrm{t} 2, \ldots, \mathrm{ts}\}$

I is an input function. I: $(\mathrm{T} \times \mathrm{P}) \rightarrow\{0,1\}, \mathrm{I}(\mathrm{t}, \mathrm{p})=1$ if there exists an arc from $\mathrm{p}$ to $\mathrm{t}$. If so, $\mathrm{p}$ is an input place for $\mathrm{t}$;

$\mathrm{O}$ is an output function. $\mathrm{O}:(\mathrm{T} \times \mathrm{P}) \rightarrow\{0,1\}, \mathrm{O}(\mathrm{t}, \mathrm{p})=1$ if there exists an arc from $\mathrm{t}$ to $\mathrm{p}$. If so,

$\mathrm{p}$ is an output place for $\mathrm{t}$;

$\mathrm{M}: \mathrm{P} \rightarrow \mathrm{N}$ is a marking that assigns a non-negative integer to each place. Such a number represents the number of tokens in the place. $<\mathrm{P}, \mathrm{T}, \mathrm{I}, \mathrm{O}, \mathrm{M}>$ represents a marked Petri net.

\section{Statement of the problem}

Pars metal, formerly known as pars Metal Pressing Enterprise is one of the manufacturing industries of the country. It was established 50 years back. The organization has passed through series of administrational structures and number of changes from time to time. It is expected to go steps forward operationally to strive for manufacturing excellence. Though more than 50 years have passed since its establishment, the company is unable to be competent and maintain its position in the market due to lack of proper management, continuous performance analysis and other various factors. The company's profit declined continuously even below the acceptable level. For this and other related 
reasons the company is forced to stop producing ammunition. Presently, by applying new management concepts and engaged in production of various spare parts, medals, badges, and furniture.

Therefore, the research work will attempt on the analysis of the manufacturing systems in the tools and spare parts work shop of the company and intend to provide solutions for its continuing problems to improve productivity and hence expanding the horizon of contribution for the development of the country .The objectives of this paper is :

- Modeling and analyzing the performance of the manufacturing process using Petri Net so as to evaluate various performance parameters such as utilization rate of machines, work-in-process, and throughput rate of system under consideration.

- Providing solutions for the pitfalls and ramification for attaining the optimum productivity.

\section{Methodology}

The methods employed to achieve the objectives of the research are:

\subsection{Data Collection}

The types of products and operations, sequence of operations for each product, the number and type of machines required and available for each product and operation, actual production time, machine down time, reasons for rejection of products and other relevant data are collected to conduct the research.Data collection was conducted by using the following methods:

- Site visiting and taking data during actual operation time,

- Conducting interviews and discussion with engineers, technicians and responsible personnel. Using previous records or documents.

\subsection{Analyzing the data by using different performance analysis techniques}

Modeling the various operations them establishes a relationship between the actual and expected performances in order to make comparison and suggest reconsiderations to be made for improving productivity of the organization under consideration.

\subsection{Case data}

In the factory there is organized data collection method for the maintenance department only.

As a result, the history of each machine, i.e. frequency and reason for failure, the time taken to repair and other relevant information is readily available for reference. Although few other

departments have trend of data collection, it is not well organized and consistent. In majority of departments including the tools and spare parts workshop the culture of data collection and

preservation is at its lowest stage. As a result, there was great difficulty to obtain relevant data.

The data required for this study such as the number and type of orders (products) with in a given period of time, the output rate of a given production line, the production schedule, machine down time, etc were gathered by personal observation, discussion with foremen, machinists and workers who have contact with the subject matter, and referring previous

documents. Below are the case data that are believed to have relevance to the work being carried out and the performance of the factory. 


\section{Modeling and performance analysis of manufacturing systems}

Modeling and performance analysis of manufacturing systems helps decision makers at higher levels to conduct an economic feasibility analysis for expansion/diversification or modification of the system. Also, this could help in installing a new manufacturing system with a substantial reduction in the number of machines, floor space, inventory level, through put and lead time and also high quality products, with a greater flexibility to respond to the market needs.

Manufacturing systems design is a complex phenomenon, which is concerned with the selection from a wide variety of available system configurations and control strategy alternatives in the light of several criteria (flexibility, quality, productivity, costs etc.), many of which are difficult to quantify. Justification and implementation of advanced manufacturing technology involve decisions that are crucial for the practitioner regarding the survival of business in the present day uncertain manufacturing world. Since advanced systems require huge capital investments and offer large number of intangible benefits such as flexibility, quality, competitiveness, customer satisfaction etc., which are ill structured in nature and sometimes very difficult to quantify[12].

\subsection{Production system modules}

In this part of the paper, a concrete step-by-step methodology is presented using PNs for modeling and performance analysis of production systems, composed of a network of workstations and buffers, where machines fail and are repaired randomly. Petri Nets (PNs) and their extensions being both a mathematical and graphical tool are widely used for modeling discrete event dynamic systems including production systems and networks. PNs have been proven to be a powerful tool for studying system concurrency, sequential, parallel, asynchronous, distributed deterministic or stochastic behavior, resource allocation, mutual exclusion and conflicts.

The major contributions of the methodology may be summarized as follows:

1. The PN based system modeling, analysis, synthesis and performance evaluation is independent of the system architecture and structure,

2. The model construction method may be extended and slightly modified in order to be applied to any configuration DEDS,

3. Analysis and synthesis of any complicated system is accomplished in terms of analysis and synthesis of the generalized PN modules, which means that the overall complexity is significantly reduced,

4. Large number of machines/ workstations could be handled, while buffer capacities may be considered either finite or infinite,

5. Whole system's analysis and properties are obtained with respect to the corresponding characteristics of the fundamental modules,

6. The generic models are simple, based in realistic assumptions and are easily applied and understandable.

A production system is usually viewed as a network of machines/workstations and buffers. Items receive an operation at each machine and wait for the next operation in a buffer with finite capacity. Random machine breakdowns disturb the production process and phenomena such as starvation and/or blocking, may occur. Due to a failed machine with operational neighbors, the level of the downstream buffer decreases, while the level of the upstream buffer increases. If the repair time is large enough, the broken machine will either block the next station or starve the previous one. This adverse effect will propagate throughout the system. 
Production control policies may be classified as token-based or time-based. Token-based systems involve token movement in the manufacturing system to trigger events. Time-based systems operate on a time basis.

The production systems concerned here in the work are non-dedicated machine production systems. In these, each machine is assigned to various operations of different product types. In such systems all parts follow the different routes. According to the proposed approach, it is possible to recognize a small number of subsystems, called from now on fundamental modules. Their corresponding Timed PN models are implemented, analyzed and used as structural components for the representation of the majority of production systems. Modules are repeated and appropriately connected in order to produce the total model of the system under review.

The use of modular subsystems in production systems modeling is a need, as this allows the independent modification of the model, results in increased flexibility that meets one of the major requirements of such systems and allows the use of more efficient advanced performance evaluation and analysis techniques, as distributed simulation [9].

\section{Petri net modules}

As already has been discussed in the preceding sections, places represent conditions; transitions represent events and arcs direct logical connection between places and transitions. PN structural and behavioral properties (reachability, safeness, conflicts, liveness, reversibility, persistency, and deadlock freeness) capture precedence relations and structural interactions between system components. Behavioral properties depend on, and are coupled with, the PN initial marking $\mathrm{m}_{0}$. Structural properties are determined using the PN topological structure following matrix-based analysis methods.

The incidence matrix $A$ for a PN consisting of $n_{p}$ places and $n_{t}$ transitions is defined as $A=\left[a_{i j}\right]$, where $\mathrm{a}_{\mathrm{ij}}=\mathrm{a}_{\mathrm{ij}}{ }^{+}-\mathrm{a}_{\mathrm{ij}}{ }^{-} ; \mathrm{a}_{\mathrm{ij}}{ }^{+}=\mathrm{w}(\mathrm{i}, \mathrm{j})$ is the arc weight from transition $\mathrm{i}$ to its output place $\mathrm{j}$ and aij $^{-}=\mathrm{w}(\mathrm{j}, \mathrm{i})$ is the arc weight to transition $i$ from its input place $j . a_{i j}{ }^{+}$, $a^{j}{ }^{j-}$ and $a_{i j}$ represent the tokens added, removed and totally changed in a place $\mathrm{j}$ by the firing of transition $\mathrm{i}$, respectively. The incidence matrix cannot represent self-loops (since the total difference of tokens in a self loop is equal to 0 ).

Dealing with time in a PN is accomplished by assigning time delays to places or transitions.Time can be associated with both nodes, but TPN models analysis is simpler when time is attached to one kind of nodes. The case described is that of PNs where time is attached to transitions, called t-timed Petri Nets. A t-timed PN arises from the corresponding Ordinary PN by associating each transition $\mathrm{t}_{\mathrm{i}}$ a firing delay that may be constant or follow a given distribution.

A TPN is defined by the tuple TPN $=\{P, T, I, O, m 0, D$,$\} such that the first five elements are as$ described above for ordinary PNs while D represents time delay and is a function from the set of nonnegative real numbers $\left\{0, R^{+}\right\} . D\left(t_{i}\right)$ is a vector whose number of elements is the same with the number of net's transitions, where $d_{i}=$ delay associated with transition $i$. A timed transition's firing consists of two events namely, "start firing" and "end firing". In between these two events the firing is in progress. Tokens are removed from input places at "start firing"' and are deposited to output places at "end of firing". The transitions delays may be deterministic or described by a distribution. In TPNs some transitions may have zero occurrence times and are called "immediate".

Figure 1. A corresponding PN modules of fundamental production systems. The PN models describe all the main events taking place in fundamental systems and are suitable for production systems simulation, analysis and performance evaluation. Modules describe common events but partially differ in operational and structural features. Timed transitions are presented as white rectangles, while immediate transitions as black. All transition input and output arc weights are equal to one. Places p0 p5 and transitions t1- t 4 have the same meaning in all four generic PNs. Transitions correspond to 
system activities resulting in state changes, while places correspond to resource (machine, parts) availability or state (machine up, down, and idle).

Table2. PN module nodes (P and T) explanation (Fig. 1)

\begin{tabular}{|c|c|c|}
\hline Node & Model & Meaning \\
\hline p0 & All four modules & Parts available in initial buffer \\
\hline $\mathrm{p} 1$ & All four modules & Machine available to process part \\
\hline p2 & All four modules & Machine breakdown \\
\hline p3 & All four modules & Parts in final buffer \\
\hline $\mathrm{p} 4$ & All four modules & Machine finished process of a part \\
\hline p5 & All four modules & Machine out of order \\
\hline \multirow{4}{*}{ p6 } & Assembly & $\begin{array}{l}\text { Second type parts available in corresponding initial buffer (raw } \\
\text { materials) }\end{array}$ \\
\hline & Disassembly & $\begin{array}{l}\text { Second type parts in the corresponding final buffer (processed } \\
\text { parts) }\end{array}$ \\
\hline & \multirow{5}{*}{$\begin{array}{l}\text { Parallel machines } \\
\text { Parallel machines } \\
\text { Parallel machines } \\
\text { Parallel machines }\end{array}$} & Second Machine (M2) available to process part \\
\hline & & Machine M2 breakdown \\
\hline $\mathrm{p} 7$ & & Machine finished process of a part \\
\hline $\mathrm{p} 8$ & & Machine M2 out of order \\
\hline p9 & & \\
\hline $\mathrm{t} 1$ & Common & Machine processing (producing) part \\
\hline $\mathrm{t} 2$ & Common & Empty machine's signal return \\
\hline $\mathrm{t} 3$ & Common & Machine breaks down \\
\hline $\mathrm{t} 4$ & Common & Machine has been repaired and is available to produce again \\
\hline $\mathrm{t} 5$ & Parallel machines & Machine M2 is processing (producing) part \\
\hline t6 & Parallel machines & Empty machine's signal return for M2 \\
\hline $\mathrm{t} 7$ & Parallel machines & Machine M2 breaks down \\
\hline $\mathrm{t} 8$ & Parallel machines & Machine M2 has been repaired and is available to produce again \\
\hline
\end{tabular}

The generic PN modules have been derived based on the realistic assumptions that: (1) buffers are finite, (2) machines operate at a given speed that changes periodically according to events taking place in the system, (3) setup and transportation times of parts through the system are negligible compared to production times, (4) machine breakdowns happen infinitely often, but only after the completion of a production cycle.

The generic transfer chain module is considered first. In this, places $\mathrm{p}_{0}$ and $\mathrm{p}_{3}$ represent the raw materials and final products buffers respectively, while $t_{1}$ describes process performance when machine is available. To avoid multiple parts reaching a machine concurrently, a signal of machine 
being empty and ready to produce is represented through a token produced from the firing of $t_{1}$ and led to $\mathrm{p}_{4}$. Empty machine's signal returns to $\mathrm{p}_{1}$, where it is initially found, meaning that next parts process may begin, following one of two possible routes. One is after firing of $t_{1}$, meaning that the next part process can begin without other events mediation. The second is after a machine breakdown represented by $\mathrm{p}_{2}, \mathrm{t}_{3}$ fires causing machine to go out of order $\left(\mathrm{p}_{5}\right)$, which is repaired after firing of $\mathrm{t}_{4}$. In all generic and generalized PN modules, conflict between $t_{2}$ and $t_{3}$ that have common input place $\mathrm{p}_{4}$, exists - machine finished a part process (in modules that have multiple machines, there exist proportional conflicts in all sets of them). To solve this conflict efficiently, $\mathrm{t}_{2}$ has been considered as a timed transition (with minimum delay) while $t_{3}$ as an immediate one. $t_{3}$ has also a second input place $\left(\mathrm{p}_{2}\right)$ (tokens are available there only in the case of a machine breakdown according to a distribution or stochastically), which means that it cannot become enabled and consequently fire if there exist no tokens in this place. So, when machine operates, timed transition $t_{2}$ fires, since immediate $t_{3}$ has no token in one input place, else $t_{3}$ fires. It must be noted that in the models presented both transitions have the same priority (equal to one), since in another case the execution of the net would be different. Generic assembly module's main difference is that two initial buffers $\left(\mathrm{p}_{0}, \mathrm{p}_{6}\right)$ provide different part types to the machine for the process to begin, while generic disassembly module has two final product buffers $\left(\mathrm{p}_{3}, \mathrm{p}_{6}\right)$. In generic parallel machine module two transfer chain PN modules are composed so that they obtain parts from the same input buffer $\left(\mathrm{p}_{0}\right)$ and lead their products to the same final buffer $\left(p_{6}\right)$. These facts are generalized for the cases of generalized PN modules. Definition of buffers maximum capacities in PN modules ensures the representation of phenomena as starved and blocked machines. Tokens shown in all four generic PN modules are for demonstration purposes only.

Observing the four generic PN modules as shown in Fig. 1 with any finite initial marking $\mathrm{m}_{0}$, one may conclude that: (1) As long as there is part availability in the input buffer(s), all four generic PNs after the completion of one production cycle return to the state of starting a new cycle; (2) the parts number in the initial buffer(s) defines the exact number of production cycles; (3) all modules are k- bounded; (4) modules are non-conservative (at least $t_{3}$ consumes two tokens and produces one, in assembly and disassembly transition $t_{1}$ is also nonconservative, in parallel machines module $t_{7}$ is non-conservative); (5) modules are nonpersistent (firing of $t_{3}$ may disable $t_{2}$ ); (6) modules are not repetitive and not consistent.

The synthesis procedure of two simplest transfer chains is shown in Fig. 3. Observing this figure, it is obvious that places $\mathrm{p}_{3}$ and $\mathrm{p}_{6}$ are fused in one place $\mathrm{p}_{3-6}$. The total number of places is reduced by one, while transitions are equal to the total of each module transitions. The combined PN input places are reduced by one ( $\mathrm{p}_{3-6}$ is an internal place, not input buffer). 
Figure 3. Synthesis of two generic transfer chain model

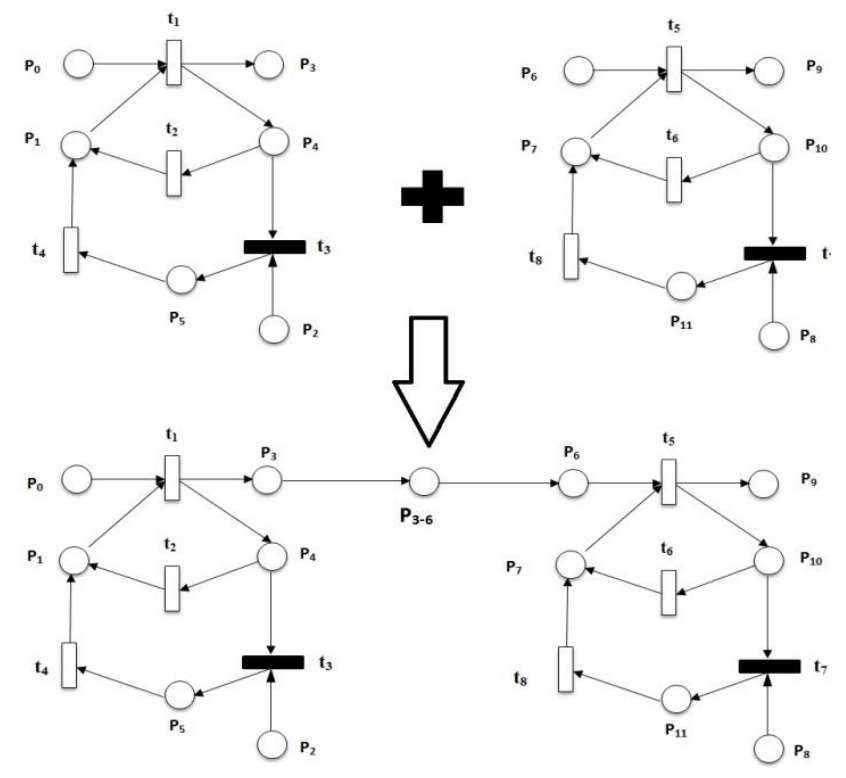

\section{Modeling of the machining processes}

The tools and spare parts workshop has 86 different types of machine tools that are used for the production purpose. This number does not include the machines that are still in the shop but broken down (those which need maintenance). There are machining stations separated by the type and capacity of the machine tools. The lathe, milling, drilling, grinding, shaping and slotting, gear shaping, jig boring, and some other machining centers are there .For performance analysis of the various processes of the shop it is necessary to model the entire shop by using the Petri net modeling, which has already been discussed in the preceding sections of this section. Fig. 4 below gives the basic model of the various production operations such as machining, heat treatment...etc.

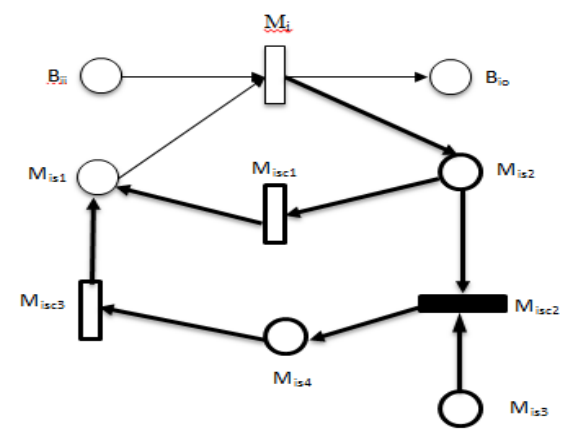

Figure 4 The basic model for different operations 
Table 3. Place and Transitions description of the basic model (Fig. 4)

\begin{tabular}{l|l}
\hline Places/transitions & Description \\
\hline Bii & $\begin{array}{l}\text { Input buffer of ith machine } \\
\text { output buffer of ith machine } \\
\text { Bio }\end{array}$ \\
Mis1 & $\begin{array}{l}\text { ith machine status 1 (available/ready for operation) } \\
\text { ith machine status 3 (machine failed) }\end{array}$ \\
Mis3 & $\begin{array}{l}\text { ith machine status 4 (machine has under repair) } \\
\text { Mis4 }\end{array}$ \\
Mi & $\begin{array}{l}\text { ith machine machine status change 1 (from un availability to } \\
\text { availability) } \\
\text { Misc1 }\end{array}$ \\
Misc2 & $\begin{array}{l}\text { ith machine status change 2 (from failure to repair) } \\
\text { Misc3 }\end{array}$ \\
\hline
\end{tabular}

Presence of tokens in places Bii and Mis1 is the pre-condition for machine Mi that initiates the operation (to be enabled and then fired). The start of operation of Mi removes one token each from the input places, Bii and Mis1, and adds it to the output place Bio and Mis2, which represents the end of operation is being performed on the part/work piece. The token in Bio represents the termination of operation on previous processor (machine) Mi and availability of finished or semi-processed part for the next process.

In the fig. 4 the bold part is dedicated for the status of the machine, i.e. updating the machine for the next operation/process and taking care of the random failure of machine.

$\mathbf{m}_{\mathbf{i}}$

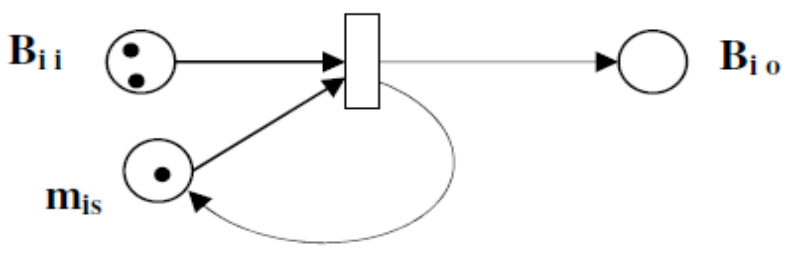

Figure 5. Simplified PNM of single machine 
Table 4 Modelling conventions with Petri nets

\begin{tabular}{l|l}
\hline Concepts in manufacturing & Petri net modeling \\
\hline Moving or production lot size & $\begin{array}{l}\text { Weight of directed arcs modeling moving or production } \\
\text { kanban } \\
\text { The number of tokens in places modeling quantity of the } \\
\text { corresponding resources }\end{array}$ \\
Capacity of work station & $\begin{array}{l}\text { The number of tokens in places modeling its availability } \\
\text { The number of tokens in places modeling the buffers and } \\
\text { operations of all machines }\end{array}$ \\
Production volume & $\begin{array}{l}\text { The number of tokens in places modeling the counter for or the } \\
\text { number of firings of transitions modeling end of a product }\end{array}$ \\
The time of an operation & $\begin{array}{l}\text { The delays associated with the place or transition modeling the } \\
\text { operation }\end{array}$ \\
time & $\begin{array}{l}\text { The delays associated with the directed arc, place or } \\
\text { transition modeling the conveyance or transportation }\end{array}$ \\
\hline Pystem state & $\begin{array}{l}\text { Petri net marking (plus the timing information for timed Petri } \\
\text { net) }\end{array}$
\end{tabular}

\section{CONCLUSIONS AND RECOMMENDATIONS}

Petri nets are being increasingly studied world wide by several researchers and industrial practitioners to address a variety of issues related to manufacturing from their specification, modeling, designing, performance analysis, and scheduling to real-time control and monitoring. However, by demonstrating the efficiencies of $\mathrm{PN}$-aided approaches to solving problems in manufacturing, industrial application of PNs can be promoted. Motivated by these facts, the present work discusses the application of PNs and proposes it as a tool and methodology for modeling performance analysis of manufacturing systems based on the previous related works, for the factory to loose its competitiveness in the market.

In this work, performance analysis is found to be the potential area for exploring both theoretical and practical application of PNs. By studying the various problems of pars metal in the stated area with PNs this work makes an attempt to analyze performance of the factory. Although the research best works in automated manufacturing area, its contribution in traditional manufacturing system like that of pars metal is significant in the study of the main factors that attribute to the said problems of the factory such as un able to meet due date, big variation between estimated and actual production/machining time, under/over cost estimation and so on. Therefore, by making use of this research work it is possible to avoid these and other related problems.

The purpose of this work is to analyze the overall manufacturing performance of the factory, indicate the key factors that contributed to the poor performance, create awareness for the need of continuous performance analysis, and finally implement or use the proposed methodology to improve the manufacturing performance by avoiding/minimizing the short comings of the factory. 
In addition to the implementation of the proposed methodology, there are many other key issues that the organization needs to revise or consider for otherwise the expected improvement or change may remain to be a nightmare. One of the issues that require serious attention and consideration is the traditional manufacturing practice of the factory, which must be changed.

Finally, the following recommendations are made based on the findings of the study:

- The factory should revise its organizational structure, must strive to be competent, and create conducive environment for improved productivity by fulfilling the necessary infrastructures.

- Process planning should be done according to its technological principle and procedure.

- Integration of various departments and workshops is also necessary.

\section{References}

[1] Haas, P J., Stochastic Petri Nets for modeling, (2004)

[2] URL: http://www.almaden.ibm.com/cs/people/peterh

[3] Kuljanic, Elso, Advanced Manufacturing Systems and Technology, SpringerWein, New York, 1999.

[4] Lakew ,Mesfin, A petri net approach to bottling line modeling and performance analysis: a case study on Meta Abo brewery Share Company, a thesis submitted to the school of graduate studies of Addis Ababa University in partial fulfillment of the requirements for the degree of Masters of Science in Mechanical Engineering, Addis Ababa University, Addis Ababa, 2004.

[5] Murata, T., "Circuit Theoretic Analysis and Synthesis of Marked Graphs," IEEE Trans. on Circuits and Systems, Vol. CAS-24, No. 7, pp. 400-405, July, 1977.

[6] Murata, T., "Petri Nets : Properties, Analysis and Applications", Processings of the IEEE, vol. 77, no. 4, 1989.

[7] Peterson, J. (1981). Petri Net Theory and the Modeling of Systems, Prentice-Hall, Inc

[8] Richardsson, J. (2005). Development and Verification of Control Systems for Flexible Automation, Licentiate thesis, Control and Automation Laboratory, Chalmers University of Technology, G“'oteborg, Sweden. Technical report 015.

[9] Sreenivas , R.S., Krogh ,B.H., "On Petri Net Models of Infinite State Supervisors," IEEE Trans. on Automatic Control, Vol. AC-37, No. 2, pp. 274-277, February, 1992.

[10] Valavanis, K.P., Modular Petri Net based Modeling, Analysis, Synthesis and Performance Evaluation of Production Systems, Journal of Intelligent Manufacturing, Vol. 16, pp. 67-92, 2005.

[11] WANG Xiao-rong, WU Tie-jun. Flexible Jobshop Scheduling Based on Petri-net Model:ACOGA Hierarchical Evolutionary Optimization Approach[J]. Journal of Zhejiang University(Engineering Science) (2004)38(3)286-291

[12] Zhang, Hehua, Gu ,Ming, Modeling job shop scheduling with batches and setup times by timed Petri nets , Mathematical and Computer Modelling 49 (2009) 286-294

[13] Zhong ,Weiping, Performance Analysis of Machining Systems with Different Configurations, Proceedings of Japan-USA Symposium on Flexible Manufacturing, Michigan, USA 2000 .

[14] Zhou, MengChu, McDermott, Kevin and Patel, Paresh A., "Petri Net Synthesis and Analysis of a Flexible Manufacturing System Cell", IEEE Trans. on Systems, Man, and Cybernetics, vol 23, no. 2, pp. 523-531, 1993 .

[15] Zhou , MengChu , Venkatesh ,Kurapati, Modeling, Simulation, and Control of flexible Manufacturing System, World Scientific, Hong Kong, 1999. 